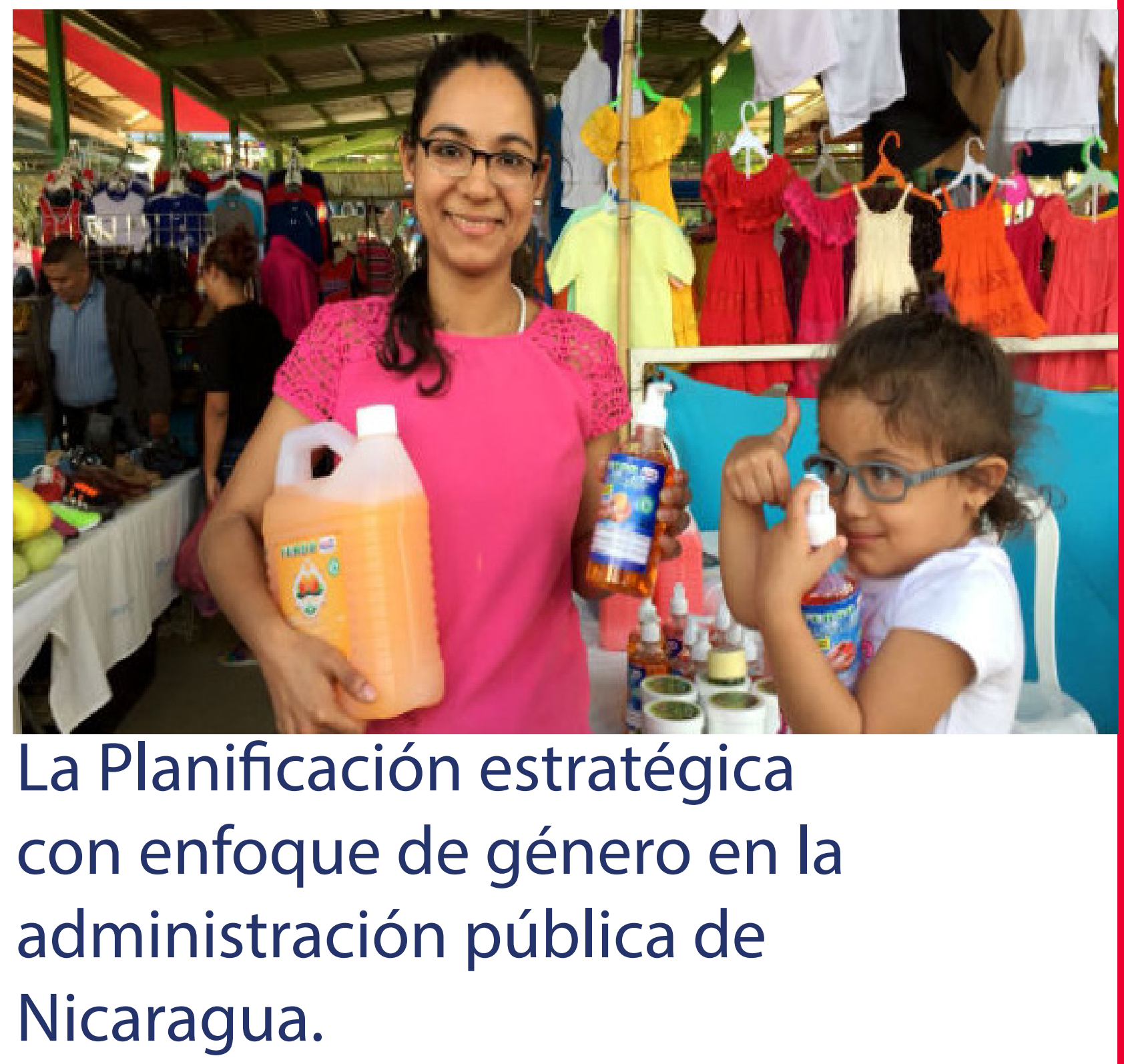

Itzel Vadivia

Politóloga y Sociología, Maestrante de Gestión y Diseño de Proyectos Sociales en la UNAN-Managua, Investigadora y Docente Universitaria y servidora pública del MHCP. https://orcid.org/0000-0002-3382-3957

valdiviaitzel04@gmail.com 


\section{La Planificación estratégica con enfoque de género en la administración pública de Nicaragua.}

\author{
Strategic planning with a gender perspective in the \\ public administration of Nicaragua
}

\section{RESUMEN}

Resumen: En las últimas décadas, con el retorno de gobiernos de izquierda en América Latina y el notable modelo cubano, se da un auge en la planificación social de modelo solidario y humanista. Nicaragua no es la excepción, con retorno del Gobierno Sandinista, en la administración pública implementa en estos 13 años, una planificación estratégica por resultado articulada con el Presupuesto General de la República con perspectiva de género, siendo la herramienta efectiva para la restitución de derechos de mujeres y hombres por medio de políticas públicas que favorezcan el desarrollo de manera integral y armoniosa con sectores sociales. Para seguir sosteniendo este modelo, se deberán superar los retos y desafíos tanto institucionales como sociales que enfrenta para fortalecer y acelerar el logro de los objetivos del desarrollo humano, que ha dado resultados de reconocimiento nacional e internacional.

\section{ABSTRACT}

In recent decades, with the return of leftist governments in Latin America and the notable Cuban model, there has been a boom in social planning of a solidarity and humanist model. Nicaragua is not the exception, with the return of the Sandinista Government, in the public administration implemented in these 13 years, a strategic planning by result articulated with the General Budget of the Republic with a gender perspective, being the effective tool for the restitution of rights of women and men through public policies that favor development in a comprehensive and harmonious manner with social sectors. To continue sustaining this model, the institutional and social challenges it faces must be overcome in order to strengthen and accelerate the achievement of human development objectives, which has yielded results of national and international recognition.

\section{PALABRAS CLAVE}

Planificación, Estratégica, Nicaragua, Desarrollo Humano, Género.

\section{KEYWORDS}

Planning, Strategic, Nicaragua, Human

Development, Gender. 
¿Me podía decir cuál es el camino que debo seguir? - preguntó Alicia. - Eso depende de dónde quieras ir - respondió el gato. - Es que no sé dónde quiero ir. —Entonces da igual el camino que tomes". priority, with migrants being part of them.

Lewis Carroll, Alicia en el País de las Maravillas, 1865.

El fin último de la planificación no es formular planes, sino cambiar positivamente la realidad, ejecutando políticas estratégicas con recursos prioritariamente dirigidos a su implementación en alineación con las políticas de igualdad de oportunidades entre mujeres y hombres. Aunque no existen "recetas" estándar, más bien un trabajo con una óptica social que supone un proceso complejo basado en el aprendizaje de nuevas formas de pensar para, a partir de ahí, "hacer aplicable” lo aprendido a cada contexto e intervención concreta.

Dentro de este orden de ideas, se podría agregar que la planificación social vinculada con los presupuestos englobados es la única alternativa ante el orden socioeconómico, cuyas leyes ciegas, ruinosas y destructivas hacen insostenible el justo desarrollo de las sociedades y la preservación de la naturaleza.

Esto lo demuestra, los trece años del Gobierno de Reconciliación y Unidad Nacional (GRUN), en la cual, se ha enfrentado enormes obstáculos y agresiones del más poderoso imperio de la historia; sin lugar a dudas, se ha superado gracias a la Planificación, caracterizada por la proliferación de estrategias y planes nacionales y multisectoriales, de manera democrática y participativa.

De la misma manera, la articulación de los planes nacionales con los departamentales y municipales, en lo formal, ha sido un propósito adecuadamente alcanzado, según lo previsto en la piedra angular de la planificación, Plan Nacional de Desarrollo Humano 2012-2016 y su continuidad Ejes del Programa Nacional de Desarrollo Humano 2018-2021. En la práctica, la ejecución de éstos -es decir la articulación real- con asignación y aplicación de recursos de las entidades públicas de los tres niveles del Estado, ha superado las expectativas esperadas en comparación con anteriores gobiernos neoliberales.

Por tanto, este ensayo pretende compartir la experiencia en implementar una planificación estratégica institucional y su articulación con el presupuesto con perspectiva de género en el Ministerio de Hacienda y Crédito Público (MHCP) durante los últimos 6 años (2013 al 2019). En él, se exponen las principales etapas fundamentales de la institucionalización de las prácticas de género, además de las características y las esferas de acción e impacto en la gestión pública a fin de beneficiar a la ciudadanía nicaragüense, así como las técnicas de análisis más frecuentemente utilizadas e instrumentos del corto, mediano y largo plazo.

Obras como estas, pretenden enriquecer la bibliografía sobre la planificación social en el sector público, donde en los últimos años, se contó básicamente con literatura de ciencias económicas, cuyos instrumentos fundamentales de análisis, aunque necesarios conocer, son aplicables, principalmente, a una economía de mercado y no a una economía socialista como la que se ha implementado por Gobierno de Izquierda como lo son Nicaragua, Ecuador, Bolivia, Argentina y Venezuela, inspirados en el modelo cubano 


\section{Consideraciones básicas sobre la Planificación}

Un plan no es nada, pero la planificación lo es todo.

Dwight D. Einsenhower

El ser humano, siempre busca escrutar el porvenir y en establecer horizontes para conducirse al mismo y a los demás; la planificación vista como una forma de actuar congénita al ser humano, continuara constituyendo un proceso imprescindible para racionalizar las decisiones y las acciones.

En el mundo profesional y gerencial contemporáneo, aún existe el debate sobre la pertinencia de la planeación para definir el norte de una organización, sea pública o privada, de forma eficiente su capital humano y material para alcanzar los objetivos trazados, en base a un análisis profundo del entorno y de la propia organización. De otro modo, la carencia de un plan estratégico rector, ha contribuido a un quehacer coyuntural, un activismo intenso y disperso dependiente de decisiones individuales, lo cual ha significado un desaprovechamiento de los recursos y una minimización de sus resultados.

Para no incurrir en lo anterior, es necesario estar claros que la planificación en términos generales es, definida por Förch (2004), como el proceso de establecer objetivos y escoger los medios más apropiados para el logro de los mismos antes de emprender la acción. Es una herramienta que permite realizar objetivos con estratégicas y acciones, define el procedimiento para su seguimiento y evaluación.

No muy distante, se plantea que la planeación estratégica es básicamente una metodología, utilizada por una entidad en donde define qué es lo que quiere alcanzar en un período determinado y los parámetros, que rigen su quehacer hacia el logro de sus resultados deseados. Para lograr esto, Berry (2007) citado por Fonseca, Hernández, Medina, et al (2014), recomienda introducir una mayor flexibilidad en la planificación estratégica, a través de: hacer énfasis en su misión; clarificar la definición de propósitos y medidas operacionales; orientarse mayormente a los resultados, a la gestión de la descentralización y a las necesidades de sus clientes (ciudadanía); hacer mayor uso de estándares de calidad y desarrollar una cultura orientada al cambio y a la innovación.

Cabe señalar que la planificación estratégica es un proceso continuo que requiere constante retroalimentación acerca de cómo están funcionando las estrategias organizacionales. Por un lado, en el sector privado, cuentan con señales de su desempeño a través de indicadores claros, tales como las utilidades, los retornos sobre la inversión, las ventas, etc. Los indicadores entregan información valiosa para la toma de decisiones respecto del curso de las estrategias, validándolas o bien mostrando la necesidad de efectuar un ajuste.

Por otro lado, lo que la ciudadanía demanda un orden y un rumbo definidos para poder desarrollar sus capacidades individuales y las de sus organizaciones, para reconocer las razones de interés público como suyas; y quieren además, buenos gestores, que alcancen los resultados esperados. Esto se debe, a que las instituciones públicas, no tienen claras las señales ni cuentan con un diseño de indicadores que permitan monitorear el curso de las estrategias, es un desafío permanente. Siendo necesaria la evaluación sobre el impacto organizacional y social que ha tenido la implementación de la planificación estratégica. 
En los últimos años, la planificación se ha enriquecido con nuevos enfoques a fin solventar las demandas ciudadanas y mejorar las condiciones de vida de las personas. Aquí es donde nace, la planificación del desarrollo considerada un contrato Estado y la sociedad, que tiene como objetivo articular las políticas públicas y su implementación en el territorio con las prioridades del país, para administrar los recursos públicos en la perspectiva de alcanzar el desarrollo sostenible.

\section{La Planificación Social en América Latina}

Los orígenes de la planificación en algunos países latinoamericanos parten de la aceptación generalizada de las resoluciones de la Conferencia de Punta del Este de 1966 con una ambiciosa lista de objetivos cuyo cumplimiento se consideraba necesario para hacer frente a los males que aquejaban a la región, se preconizaba la necesidad de planificar el desarrollo; también, intenta desacreditar la Revolución Cuba de 1959.

En contraste con ello, Granadillo \& Vigoa (2008:21), explican que la planificación en Cuba se concibió desde inicios de la Revolución no sólo como un mecanismo económico, sino que se identificó como la categoría distintiva del Socialismo. Hasta nuestros días, ha sido un elemento central en el sistema de dirección de la economía y le ha permitido al Estado cumplir las directrices de la política económica acordada al ejercer las funciones de coordinación, asignación, redistribución, regulación y control de los recursos necesarios para asegurar la estrategia de desarrollo trazada.

En la obra de Leiva Lavalle, Jorge. (2012), detalla la transición del paradigma de la Planificación en el sector público en los años venideros; en los años 70's, la planificación entro en un período de transición caracterizado por el declive del modelo estatista industrializador, el proceso de internalización financiera por Betton Woods en 1971, y del desarrollo de los mercados cambiarios y de los eurodólares depuestos del 1973, además de una etapa de dictaduras de derecha en la Región.

En los años 80's comenzó la crisis de la deuda externa iniciada y las revoluciones sociales en Nicaragua y El Salvador; para los 90's, los programas de reforma estructural inspiradas en el Consenso de Washington redujeron la actividad e influencia de la planificación en la gestión pública, desarticulándola de la presupuestación. Como bien, se observa el retroceso de la Región por las políticas neoliberales.

Mientras tanto Cuba, afirma Granadillo \& Vigoa (2008:41-42), siguió fortaleciéndose en el campo de la planificación, fundamentalmente sobre un mayor papel del plan en la economía nacional, la planificación empresarial, las inversiones y la participación más activa de los trabajadores en el proceso de análisis y aprobación del plan. Se introduce la planificación por programas dirigida a una mayor integralidad en las proyecciones de las diferentes entidades que contribuyen a un objetivo priorizado.

Las últimas décadas atestiguan un notable auge de diversas actividades desarrolladas en tomo al tema de la planificación nacional; con el éxito del modelo cubano en la planificación y al retorno de gobierno socialistas durante el nuevo milenio, se proyecta el modelo solidario y humanista del ALBA, liderado por Venezuela y en muchos países se rescatan lo mejor de las experiencias en la planificación. 
En el 2008, se realiza en Quito, la primera Reunión de Ministros de Planificación de América Latina, en la que consistía en el intercambio sostenido fueron examinados las experiencias de unos y otros, se expresó el deseo de establecer una fluida comunicación e intercambio de información y documentación entre nuestras instituciones, a lo cual este libro modestamente también puede contribuir.

Con los gobiernos del Buen Vivir, nace el interés en que las instituciones públicas, abandonen la ineficacia y lentitud, ineficiencia, falta de reflejos para atender las nuevas demandas sociales, incapacidad de contener el gasto público, y relaciones de naturaleza burocrática con la ciudadanía, para buscar mayor productividad, imaginación e innovación.

Hay que mencionar, además, que aparecieron nuevos conceptos y otros retomaron bríos en ese particular momento histórico: desarrollo humano, bienestar, capital social, estrategias de supervivencia, buen vivir, restitución de derechos, y principios como la dignidad, soberanía, igualdad y equidad. Todas dirigidas a analizar, interpretar y eventualmente compensar la situación de una masa creciente población vulnerable y en estado de desigualdad social.

Por tanto, los Gobiernos de Izquierda se plantearon acciones sobre dos aspectos transversales, en primer lugar, los contenidos de sus agendas políticas orientadas a quebrar los postulados centrales del Consenso de Washington, que a fin de cuentas no hacía más que recoger, aquella fatídica lista de diez políticas, adoptada como un dogma de fe por los organismos financieros internacionales, y que posteriormente fue impuesto a sangre y fuego en los países del llamado tercer mundo, tras el fin de la Guerra Fría y en segundo lugar, la recuperación de las capacidades estatales.

En este sentido, el Comandante Hugo Chávez en 1996, dio a conocer "la Agenda Alternativa Bolivariana", el plan de acciones con un enfoque humanístico, integral, holístico y ecológico, al cual definió, en sus propias palabras, como "un arma para la contraofensiva total". Este se trataba de la respuesta a la aplicación del paquete de medidas económicas de corte neoliberal bautizado como “Agenda Venezuela", en marcha desde abril de 1996, el cual no podría ser considerado un plan o programa, pues consistió básicamente en una lista o "agenda" de exigencias del Fondo Monetario Internacional para conceder empréstitos al gobierno del entonces presidente Rafael Caldera.

Así pues, se empezaron a formaron caminos alternativos para fortalecer y mejorar la gestión estatal en responsabilidades centrales que garanticen la cohesión social y recuperen el lugar de la política como instrumento de construcción de futuro.

Un caso notable es Bolivia con el Presidente Evo Morales Ayma, en la cual planteó, el Plan Nacional de Desarrollo de Bolivia 2006-2010, posteriormente el Plan Nacional de Desarrollo 2010-2015 del programa del MAS y, por último, la Agenda Patriótica 2025, a fin de constituir una sociedad y un Estado más incluyente, participativo, democrático, sin discriminación, racismo, odio, ni división.

Otro país del cono sur, que presentó Plan Nacional de Desarrollo fue Ecuador para el 2007-2010, consecutivamente Plan Nacional para el Buen Vivir de Ecuador 2009-2013, que lo denomina como una “Plan para la Revolución Ciudadana", cuya propuesta, no se aleja de lo planteado por Venezuela, Bolivia y por supuesto de Nicaragua, estos fueron partes de intercambios que aportan al pensamiento latinoamericano sobre los procesos de descolonización en las políticas públicas, a través de la recuperación y visibilizarían de formas de conocimiento que incluyen realidades suprimidas, marginadas y/o excluidas, a través de los diferentes organismos integradores como el ALBA y MERCOSUR. 
Precisamente, la planificación en Nicaragua contó desde sus orígenes, en 1969, con la dirección y orientaciones del Frente Sandinista de Liberación Nacional, El Programa Histórico del Frente Sandinista de Liberación Nacional (FSLN) conocido como la Herencia Programática de Sandino, es el reflejo de la interpretación de la realidad social de Nicaragua y la exposición de los postulados fundamentales que guiarían las transformaciones revolucionarias, una vez que el régimen somocista fuera liquidado y el poder del pueblo se convirtiera en realidad. A pesar, que no tenía una legalidad jurídica por el contexto de la Dictadura Somocista; si tenía validez para la población nicaragüense. Caso contrario, fue la Ley No. 7 Ley de Planificación Nacional, aprobada el 27 de diciembre de 1974; y no se registra literatura al respecto sobre su implementación y logro en la gestión pública.

Durante los 16 años de gobierno neoliberales, se ubican dos instrumentos de planificación; en el Período de Enrique Bolaños (2002-2007), el primero “Estrategia Reforzada de Crecimiento Económico y Reducción de Pobreza (ERCERP)", y, en el 2005 "El Plan Nacional de Desarrollo (PND) ambos consistieron en ajuste estructural y disciplina fiscal e incentivos a las empresas privadas para crecimiento macroeconómico, sin invertir en la población más vulnerable del país.

A partir del 2007, con el retorno del Gobierno de Reconciliación y Unidad Nacional -GRUN e inspirado El Programa Histórico del FSLN, se orientan políticas públicas enfocadas en impulsar el crecimiento económico y el desarrollo social de una forma sostenible y equilibrada. Al mismo tiempo, la Secretaría de la Presidencia (SEPRES) es la encargada de crear una Matriz Directiva del PNDH donde se sintetizan lineamientos, indicadores, ejes y metas del ONDH para una exitosa implementación del Plan. Por último, el CONPES elabora el presupuesto a partir de Marcos Presupuestarios de Mediano Plazo que se armoniza con el PNDH y en sus aspectos macroeconómicos con el Programa Económico Financiero (PEF).

La agenda pública nacional, está definida en el Plan de Desarrollo Humano 2012-2016 y la continuidad, los Ejes Nacional de Desarrollo Humano 20182021 en congruencia con el lema de la Agenda 2030, “Nadie se queda atrás". Vale decir, que el PNDH es entendido como un proceso sistemático de ejecución de políticas, planes y programas, acompañadas de transformaciones profundas del rol de Estado y de sus instituciones, principalmente de sus incidencias en la dinámica de modelo económico y social, tal como se describe a continuación.

De esta manera, se integra enfoques de políticas públicas en diferentes ámbitos; como la promoción de un ambiente atractivo para los inversionistas, caracterizado principalmente por la estabilidad de precios, el repunte de las principales actividades económicas, la diversificación de la oferta exportable, la sostenibilidad de las finanzas públicas y la robustez del sistema financiero.

En el ámbito social, se ha mostrado importantes avances en indicadores como esperanza de vida y el nivel de escolaridad; $y$ en términos generales ha avanzado gradualmente en la reducción de los indicadores de pobreza nacional y pobreza extrema. Los avances en materia económica han obtenido el reconocimiento de organismos multilaterales y de las agencias calificadoras de riesgo. 
A juicio del politólogo, Cairo Amador, en una entrevista realizada en el 2018 por el19digital, los programas socioeconómicos que desarrolla el Buen Gobierno Sandinista, son fundamentales para impulsar los valores, la integridad y la unidad familiar en Nicaragua. En este sentido, también valoró que el Programa Calles para El Pueblo, dignifica las condiciones de vida donde las familias se desarrollan, y de esta manera tienen mayores oportunidades para crecer en paz y prosperidad.

De igual manera, se les ha restituido los derechos y empoderamiento de sectores históricamente discriminados en Nicaragua; tal como el caso de la juventud que actualmente, se sientes respaldados y en condiciones para un desarrollo integral, así lo demuestran las cifras presentadas el pasado 8 de septiembre del presente año, en la cual, se cuenta con 3,446,640, jóvenes de ambos sexos con participación protagónica, llevando alimentos, trabajo voluntario y diversas actividades de carácter social, así lo publica, el19digital.

Referente a programas sobre empoderamiento de la mujer, se ha trabajo en tres ejes; primer lugar en el fortalecimiento de capacidades, capitalización y organización de dos programas emblemáticos, Hambre Cero y Usura Cero, siendo beneficiadas 198.693 mujeres y 347,835 para el segundo programa. Además del reconocimiento internacional, al posicionar en 5to lugar en el índice de Brecha Genero 2020; y en 3er lugar, en el informe de "mujeres en la Política" de ONUMUJERES. En referencia al acceso de la justica, se reanudan las Comisarias de la Mujer, así como el fortalecimiento del marco jurídico - político en igualdad de género y protección hacia mujeres.

Otro punto importante, es el reconocimiento de tierras comunales, por lo cual se les garantiza protección especial, por su naturaleza inembargable, imprescriptible e intransferible. Además, se cuenta una estrategia construida desde y por los propios pueblos de la Costa Caribe, promoviendo esa conectividad con el resto del País.

Para que la implementación de una política pública se materialice, requiere planificar y organizar el aparato administrativo del Estado y de otros cestos intervinientes, y asegurar la disponibilidad de recursos materiales, humanos, económicos- financieros y tecnológicos, tal como lo ha hecho el Ministerio de Hacienda y Crédito Público, dando como resultados el reconocimiento del representante del Banco Mundial en Nicaragua, Luis Constantino (el19digital.com; 1/2/2018) por los altos niveles de ejecución de diferentes programas, como los que se desarrollan con fondos del Banco Mundial. Detrás de estos logros y avances, se encuentra un trabajo de articulación interinstitucional; así lo reafirma, Goodstein, Nolan \& Pfeiffer (1998), la única razón válida para la planificación estratégica consiste en lograr su implementación diaria.

En caso específico, El Ministerio de Hacienda y Crédito Público, conforme al siguiente marco jurídico; Ley No. 290, "Ley de Organización, Competencias y Procedimientos del Poder Ejecutivo", publicada en La Gaceta, Diario Oficial №35, del viernes 22 de febrero 2013, Arto. 21 Atribuciones del Ministerio de Hacienda y Crédito Público; Ley No.550, "Ley de Administración Financiera y del Régimen Presupuestario", publicada en La Gaceta No.167 del 29 de agosto de 2005, Artos. 153, 154 y 156.

Las leyes conexas como Ley No. 648 "Ley de Igualdad de Derechos y Oportunidades" que en el capítulo II Ámbito Económico, articulo No. 14 y 15 establece que las instituciones deben asignar partidas presupuestarias para las prácticas de género. Asimismo, en el capítulo III Disposiciones Generales, Articulo No.6 orienta realizar los ajustes necesarios en su Política Presupuestaria para el respectivo funcionamiento de las acciones encaminadas al cumplimiento del enfoque de género en las políticas públicas de todos sus órganos administrativos. Además, del marco normativo legal, está el político, tal como la Política de Género del Gobierno de Reconciliación y Unidad Nacional, GRUN, junio 2007. 
Con base en el marco legal, el MHCP tiene la visión de "Ser una institución líder, modernizada y fortalecida que garantice una política fiscal en apoyo a la estabilidad macroeconómica, en un marco de desarrollo integral de recurso humano, garantizando prácticas de género en la gestión institucional con procesos eficientes y prestación de servicios de calidad, para contribuir al cumplimiento de las políticas y programas estratégicos establecidos en el Plan Nacional de Desarrollo Humano (PNDH)."; para cumplir con está, tiene como misión de "Rectorar las finanzas públicas en apoyo a las políticas nacionales dirigidas al crecimiento económico y a la reducción de la pobreza, conforme las prioridades establecidas en el Plan Nacional de Desarrollo Humano".

Desde el 2007, el Ministerio de Hacienda y Crédito Público (MHCP) ha encaminado una Planificación Estratégica con Prácticas de Género en la planificación y proceso de presupuestario; en cumplimiento a los lineamientos del primer Plan Estratégico 2011-2015 y posteriormente el Plan Estratégico 2016-2020, esta vez desde un enfoque por resultado, perspectiva de género y gestión de riesgo, ambos inspirados en el PNDH 2012-2016.

Con estos antecedentes, se inicia una nueva etapa de la Planificación Institucional Estratégica, que facilita la articulación planificación -presupuesto por medio de indicadores institucionales del Marco de Gasto Institucional de Mediano Plazo (MGIMP) y el Sistema de información del Gobierno de Reconciliación y Unidad Nacional (SIGRUN).

Esta articulación se da con las Normas y Procedimientos de Ejecución y Control Presupuestario que se publican cada año en concordancia con la Metodología para la incorporar prácticas de género en el PGR y MGIMP. Por ejemplo, para el 2020, en el acápite Responsabilidades del Registro de la Ejecución, en el art 45. “Las Dirección Generales de Planificación, registrarán la ejecución física, de los indicadores definidos en el Presupuesto 2020..."

Sobre la misma idea, se desea analizar cómo concibe el GRUN-en este caso, el Ministerio de Hacienda y Crédito Público, - las cuestiones de género a la hora de ejercer políticas dirigidas a instituciones donde todavía se constata la existencia de relaciones de género, forjando a su vez dispositivos comunicativos e informativos de dichas políticas en los que se ve representado, a través de imágenes o discursos, el imaginario genérico propuesto desde el Estado.

Este último, nace en el 2012 por los esfuerzos del GRUN y la Entidad de las Naciones Unidas para la Igualdad de Género y el Empoderamiento de las Mujeres (ONU-MUJERES) para ejecutar el Programa "Incremento de la Responsabilidad en la Financiación para la Igualdad de Género-F4GE", el cual se ejecutó por el MININ, MAG, MHCP, MINSA, MIFAN, MIGOB, MIFAN, MINREX y Asamblea Nacional.

Para el desarrollo del Plan Estratégico por resultado, perspectiva de género y gestión de riesgo 2016-2020 del MHCP, está organizado en cinco objetivos que responde a la estructura funcional y orientada a respalda la operacionalización efectiva de las políticas y objetivos del PNDH. Y cada objetivo apuntan a resultados estratégicos e indicadores cuantitativos y cualitativos respectivos, que representa la síntesis del conjunto de acciones identificas por las unidades administrativas; se trabaja una Metodología de Planificación por Resultado con Prácticas de Género, que estable la aplicación de criterios de género como el vocabulario inclusivo, información desagregada por sexo y el marco normativo de género. A lo interno del MHCP, las 22 unidades administrativas presentan un Plan Operativo Anual (POA) y que es monitoreado de forma mensual y trimestral. Este proceso es conducido por la División de Planificación (DP) y la Unidad Técnica de Género (UTG). 
Tal como se muestra, la naturaleza estratégica de la planificación radica en su capacidad de visualizar el cambio necesario y posible de la situación, en la medida en que se analizan oportunidades y riesgos que inciden en el ámbito de acción. Este análisis tiene como fin generar estrategias para mejorar la viabilidad de la propuesta de cambio, por lo que la Planificación Institucional Estratégica, se convierte en un instrumento idóneo que implica el conjunto de decisiones que preceden y presiden la acción; su carácter estratégico en el mediano y largo plazo puede anticipar un cambio social real, dada la complejidad que tiene todo sistema social.

\section{Conclusión}

El análisis realizado en las páginas precedentes permitiría inferir algunas conclusiones:

Se ha demostrado que la planificación estratégica proporciona beneficios a todas las organizaciones, independientemente de su tamaño o experiencia. Para que la entidad pueda obtener el mayor rendimiento posible, necesita una planificación estratégica eficaz que le permita definir con precisión sus objetivos y políticas, y que la conduzca hacia una mejor coordinación de esfuerzos. De hecho, el plan estratégico implica una adaptación de la organización a las oportunidades de un entorno en permanente cambio, adaptando las acciones actuales para un futuro posterior y aprovechando al máximo sus recursos internos que le confieren ventaja con respecto a la competencia.

Teniendo en cuanta que la Planificación Institucional responde a un marco de planificación superior definido normativamente por el Comisión Nacional de Planificación Económica y Social (CONPES). No se desarrolla en el vacío, sino que responde y debe articularse a las políticas públicas de mayor nivel definidas en los planes de desarrollo y en los planes sectoriales. Cada institución, al momento de formular su "intención estratégica", es decir, de llevar adelante su Planificación Institucional que se plasma en el PE 2015-2020, debe identificar y definir el marco de planificación superior al que debe responder.

Cabe señalar, que una de las prioridades del PNDH 2018-2020 es contribuir a disminuir la situación de discriminación e inequidad y crear posibilidades de acceso a las oportunidades y beneficios, para la cual es necesario que el sector público, tome las mejores decisiones y el compromiso de una sociedad más justa. Aquí, la planificación con enfoque de género es una herramienta de primera mano para facilitar la incorporación de los intereses de mujeres y hombres en sus propios procesos de desarrollo.

En cuanto a los retos de la planificación estratégica institucional con enfoque de género en el sector público son múltiples, comenzando que, este modelo implica superar la cultura del cortoplacismo y la improvisación, además el método participativo con enfoque de género plantea un desafío a la tradición centralista y machista que predomina en la sociedad nicaragüense.

En definitiva, para la realización exitosa de un proceso de planeación estratégica requiere al menos dos condiciones básicas, en primer lugar, una conciencia de su valor considerando que se trata de una inversión tan importante como la infraestructura física, sumando a una voluntad política y colectiva de ejecutarla en tiempo y forma. En segundo lugar, se requiere de herramientas metodológicas sencillas, productivas y de validez comprobada, tal como nos ofrece la experiencia del MHCP. 


\section{Referencias bibliográficas.}

- Cooperación Técnica Alemana-GTZ. (2009). Planificación Institucional: Conceptos claves e instrumentos Metodológicos utilizados en los Procesos de Asesoramiento de la Cooperación Técnica Alemana. La Paz, Bolivia.

- De Mattos, Carlos A. (1979). Planes versus planificación en la experiencia Latinoamérica. Revista de la CEPAL. Agosto, 1979. P. 79 - 96. Perú.

- $\quad$ Förch, Michael. (2004). La Gestión del futuro: El modelo de la Planificación estratégica participativa con perspectiva de género. $1^{1}$. Edición. HISPAMER. Managua.

- $\quad$ Fonseca. Joao Pedro Da-, Hernández-Nariño, Arialys Medina-León. Alberto, Nogueira-Rivera, Dianelys. (2014: 105-112). Relevancia de la planificación estratégica en la gestión pública. Ingeniería Industrial/ISSN 1815-5936/Vol. XXXIV/No. 1/enero -abril/2014/p. 105-112.

- Granadillo, Mildrey \& Vigoa, Claudio. (2008). Herramientas Básicas del Planificador. Centro de Estudios de Economía y Planificación (CEEP). Ministerio de Economía y Planificación. Montevideo, Uruguay.

- Gobierno de Reconciliación y Unidad Nacional -GRUN. (08 noviembre 2012). Plan Nacional de Desarrollo Humano 2012-2016. Nicaragua.

- Gobierno de Reconciliación y Unidad Nacional -GRUN. (diciembre 2017). Ejes del Programa Nacional de Desarrollo Humano 2018-2021. Nicaragua.

- Poveda Gómez, Abdón Alejandro. (2008). Planeación del Desarrollo. Programa Administración Pública Territorial. Escuela Superior de Administración Pública. Bogotá.

- Leiva Lavalle, Jorge. (2012). Pensamiento y Práctica de la Planificación en América Latina. Instituto Latinoamericano y del Caribe de Planificación Económica y Social (ILPES), Chile.

Itzel Vadivia

Politóloga y Sociología, Maestrante de Gestión y Diseño de Proyectos Sociales en la UNAN-Managua, Investigadora y Docente Universitaria y servidora pública del MHCP. https://orcid.org/0000-0002-3382-3957 valdiviaitzel04@gmail.com 